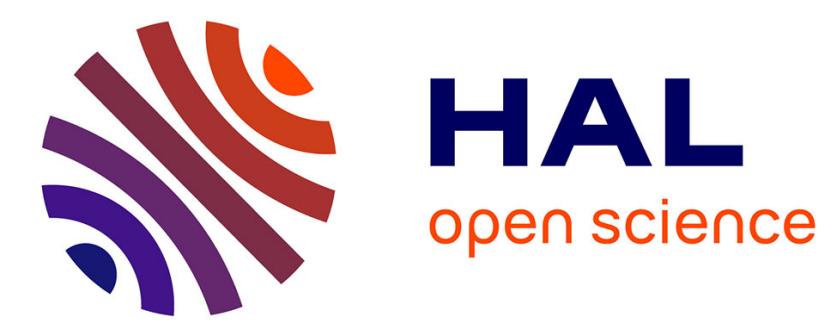

\title{
Le monument violenté : de l'émotion à la mise en exposition
}

Isabelle Brianso

\section{To cite this version:}

Isabelle Brianso. Le monument violenté : de l'émotion à la mise en exposition. Culture et Musées, 2020, 36, pp.35-56. 10.4000/culturemusees.5391 . hal-03041715

\section{HAL Id: hal-03041715 https://hal.science/hal-03041715}

Submitted on 29 May 2021

HAL is a multi-disciplinary open access archive for the deposit and dissemination of scientific research documents, whether they are published or not. The documents may come from teaching and research institutions in France or abroad, or from public or private research centers.
L'archive ouverte pluridisciplinaire HAL, est destinée au dépôt et à la diffusion de documents scientifiques de niveau recherche, publiés ou non, émanant des établissements d'enseignement et de recherche français ou étrangers, des laboratoires publics ou privés. 


\section{OpenEdition}

Journals

\section{Culture \& Musées}

Muséologie et recherches sur la culture

$36 \mid 2020$

L'émotion dans les expositions

Dossier

\section{Le monument violenté : de l'émotion à la mise en exposition}

The assaulted monument: from emotion to exhibition

El monumento violentado: de la emoción a la exhibición

\section{ISABELLE BRIANSO}

p. $35-56$

https://doi.org/10.4000/culturemusees.5391

\section{Résumés}

Français English Español

Lorsqu'un monument est violenté par les forces armées ou les milices locales lors d'un conflit armé, il change instantanément de statut : il devient une ruine traumatique (Leblanc, 2010). Cette " mise à mort » produit une émotion patrimoniale " négative » (indignation, sidération ou choc) qui circule dans l'arène sociale grâce à une pluralité d'acteurs (habitants, experts, etc.). Les violences infligées au patrimoine antique du Moyen-Orient ont été le support discursif de l'exposition immersive Cités millénaires. Voyage virtuel de Palmyre à Mossoul présentée à l'Institut du monde arabe en 2018-2019. Notre propos est d'étudier la fabrique émotionnelle du monument violenté ainsi que les stratégies expographiques mobilisées pour déclencher l'émotion chez le visiteur dans son parcours de visite. Notre approche méthodologique s'appuie tant sur l'analyse sémiotique d'effets scéniques du monument en ruine dans les salles d'exposition (image, film) comme expérience empathique pour les visiteurs, que sur l'étude des stratégies numériques à l'œuvre (reconstitution $3 \mathrm{D}$ ) et des récits intimes d'acteurs engagés face aux monuments violentés.

When a monument is assaulted by armed forces or local militia within the context of an armed conflict, it immediately changes status, becoming a traumatic ruin (Leblanc, 2010). This "execution" produces a "negative" heritage sentiment (indignation, disbelief or shock) which circulates in the social arena thanks to a number of different sources (inhabitants, experts, etc). The damage inflicted upon ancient Middle Eastern heritage sites was the basis for the immersive exhibition Cités Millénaires. Voyage virtuel de Palmyre à Mossoul (Age Old Cities. A Virtual Journey from Palmyra to Mosul) at the Institut du monde arabe in 2018-2019. This article proposes a study of the emotional charge of the assaulted monument as well as of the expographic strategies employed in order to create an emotional reaction on the part of the visitor during their visit. Our methodological approach consists of both a semiotic analysis of the staging effects of the ruined monuments in the rooms of the exhibition (through images and films) as an empathic experience for visitors, and a study of the digital strategies deployed (3D reconstitutions) and of the personal stories of those committed to the assaulted monuments. 
Cuando un monumento es violentado por las fuerzas armadas locales o las milicias durante un conflicto armado, su estatus cambia instantáneamente: se convierte en una ruina traumática (Leblanc, 2010). Esta «ejecución» produce una emoción patrimonial «negativa» (indignación, asombro o conmoción) que circula en el ámbito social gracias a una pluralidad de actores (habitantes, expertos, etc.). La violencia infligida al patrimonio antiguo de Medio Oriente constituyó el soporte discursivo de la exposición inmersiva Ciudades Milenarias. Viaje virtual de Palmira a Mosul presentado en el Instituto del Mundo Árabe en el 2018-2019. Nuestro objetivo es estudiar la construcción emocional del monumento violentado, así como las estrategias expográficas movilizadas para provocar las emociones del visitante durante su itinerario de visita. Nuestro enfoque metodológico se basa tanto en el análisis semiótico de los efectos escénicos del monumento en ruinas, en las salas de exposición (imagen, película) como una experiencia empática para los visitantes, como en en el estudio de las estrategias digitales (reconstrucción 3D) y las narraciones íntimas de los actores involucrados en los monumentos violentados.

\section{Entrées d'index}

Mots-clés : monument violenté, émotion patrimoniale, ruine traumatique, acteurs, exposition

Keywords: damaged monuments, heritage emotion, traumatic ruin, actors, exhibition

Palabras clave: monumento violentado, emoción patrimonial, ruina traumática, actores, exposición

\section{Notes de la rédaction}

Manuscrit reçu le 24 juillet 2019

Version révisée reçue le 15 avril 2020

Article accepté pour publication le 3 juin 2020

\section{Texte intégral}

\section{Introduction}

Depuis la destruction des bouddhas de Bâmiyân par les talibans (2001), les monuments et les sites archéologiques antiques du Moyen-Orient sont régulièrement pris en otage dans les conflits armés (guerre, terrorisme), voire défigurés ou sacrifiés par les forces militaires et les milices locales. Élevés au rang de "sites martyrs ", ces derniers sont instrumentalisés par les terroristes comme trophée idéologique et médiatique suscitant indignation et émotion de la part de l'opinion publique et des institutions politiques. L'Unesco dresse ainsi, quasi impuissante, l'inventaire de ces ruines archéologiques dont elle essaie de redessiner tant bien que mal les traces matérielles à partir de plans d'architecture, d'archives historiques ou d'images virtuelles. Dans ce contexte, le monument se présente en tant que « substrat sensible » (Rautenberg, 2008 ; Riegl, 1984), sorte d'être animé (habitant, ancêtre) témoignant in situ des sévices physiques de la guerre (Kott, 2013 ; Leblanc, 2010), comme le soulignait dès 1914 Albert Londres en comparant la cathédrale de Reims impactée de 287 marques d'obus à un soldat qui « vous montrerait sa poitrine déchirée ». Dès lors, une empathie patrimoniale (Waty, 2016) se dessine vis-à-vis du monument " agressé », « violé », « condamné », « pillé », « violenté » ou « mutilé » par un tiers (militaire, soldat, terroriste), et qui semble bouleverser les témoins de ce chaos. En effet, cette déferlante brutale de violence sidère les habitants locaux et choque l'Occident, induisant ainsi la construction d'une émotion collective à l'égard de ces ruines " traumatiques » (Leblanc, 2010) jusqu'à pousser certains à une mobilisation " en faveur du patrimoine, la plupart du temps pour son maintien à l'identique, et qui est nourrie par différents types d'émotions » (Kott, 2013 : 120). Notre propos ici est d'examiner, dans un premier temps, la notion de monument pris dans un processus de destruction volontaire en situation de guerre comme arène propice à l'émotion : qu'estce qui caractérise un monument meurtri ou détruit par la guerre ? Pourquoi est-il un vecteur émotionnel fort ? Quels sont les mécanismes de cette fabrique émotionnelle? Puis, nous tentons d'analyser comment une vive émotion patrimoniale suscitée par un 
conflit armé a été le prétexte à une exposition immersive présentée à l'Institut du monde arabe (IMA), Cités millénaires. Voyage virtuel de Palmyre à Mossoul. Nous questionnons les stratégies expographiques mobilisées pour susciter l'émotion chez les visiteurs (reconstitution $3 \mathrm{D}$, ambiance urbaine), et les types d'acteurs mis en scène dans l'exposition (habitants, experts) pour interpeler le visiteur au cours de son expérience de visite (livre d'or). L'exemple des salles consacrées aux violences, matérielles et mémorielles, infligées au site archéologique de Palmyre par l'État islamique nous a semblé être un choix pertinent pour illustrer la notion de monument violenté en tant que " trace traumatique » qui mobilise des émotions patrimoniales dites « négatives » au sens de Heinich $(2012,2013)$.

\section{Le monument violenté : une arène à émotions?}

L'histoire millénaire de l'humanité s'est construite autour de civilisations fleurissantes, de jeux de pouvoir, mais aussi de tensions qui ont conduit de grands centres urbains antiques à des périodes plus sombres de leur développement, engendrant déclin, asservissement, voire disparition complète. Le monument se présente en Occident en tant que trace visible de ces civilisations disparues dont les formes et les représentations ont évolué au fil des siècles, des flux artistiques et des zones géographiques. Depuis l'Occident, le monument incarne dans l'imaginaire collectif cette trace physique d'une civilisation antique disparue ; il convient néanmoins de rappeler que le monument en tant qu'objet patrimonial a connu une « transmutation lente », notamment en France, en raison de son « appartenance [...] à la culture d'élite de la société d'Ancien Régime » (Andrieux \& Chevallier, 2014 : 9). Ainsi, un monument peut être qualifié comme un " édifice imposant par sa taille et remarquable par son intérêt historique ou esthétique, par sa valeur religieuse ou symbolique ${ }^{1}$ ». Cette définition amène à distinguer le monument « isolé » et le monument appartenant à un ensemble (site) dont les éléments patrimoniaux favorisent l'interprétation des fonctions, usages et significations. Selon Jean Davallon (2015), l'étude des monuments relève d'une double origine dès lors qu'ils sont conservés, exposés et transmis par les institutions habilitées à les protéger. En effet, le monument en tant qu'objet patrimonial reconnu comme tel en Occident est, certes, un « objet matériel, mais aussi fiches descriptives, enregistrements, comptes rendus de recherche, catalogues, ouvrages, etc. » (ibid.). Cette " patrimonialisation productrice du savoir », au-delà de l'objet matériel, transforme le statut de l'objet grâce à une étude savante de ce dernier (contexte, dimensions, esthétique, etc.) afin de " reconstituer la mémoire perdue de l'objet et de son monde d'origine, de son contexte de production » (ibid.). Le monument se présente, dès lors, non plus en tant que simple trace authentique du passé, mais bien comme un objet "vivant » doté d'une mémoire devant être documentée, car productrice de savoirs. Le monument s'apparente donc à un objet aux fondements complexes et sensibles, au-delà de sa seule matérialité, du fait qu'il garde la mémoire du groupe (famille, société, habitants). En somme, il « n'est plus une carcasse, c'est un cœur battant », comme le souligne Philippe Bélaval, président du Centre des monuments nationaux, qui s'inscrit durablement dans le quotidien spatial et culturel des acteurs du groupe afin de " contribuer à maintenir et préserver l'identité d'une communauté, ethnique ou religieuse, nationale, tribale ou familiale » (Choay, 1996 : 15). Par ailleurs, le monument symbolise le bien culturel universel par excellence. Il est doté d'une valeur d'ancienneté, mais aussi historique, sociale, esthétique et d'authenticité comme le rappellent les spécialistes en sciences humaines et sociales qui ont questionné dans leurs travaux la notion de «valeur » associée au monument (Choay, 1996 ; Davallon, 2006 ; Heinich, 2013 ; Riegl, 1984). C'est pourquoi, lorsqu'il est menacé par des éléments endogènes ou exogènes liés à son environnement proche ou à des conflits armés, la perte partielle ou totale du bien contribue à la construction d'un foyer émotionnel que nous souhaitons analyser. Bérénice Waty propose une typologie de trois circonstances exceptionnelles qui seraient propices aux émotions 
patrimoniales et qu'elle définit comme des «mobilisations populaires en faveur d'un bien patrimonial » (2016:15). Selon elle, la première situation serait liée aux accidents causés volontairement ou involontairement par l'homme (sinistre, incendie, dégât des eaux, etc.). La seconde circonstance interviendrait lors d'une catastrophe naturelle, mais dont les causes ne sont pas directement liées à l'intervention humaine, plutôt en lien avec des phénomènes naturels majeurs (crue, tsunami, tempête, foudre, etc.). Le troisième cas concernerait les guerres et les conflits armés en tant que destruction volontaire à l'égard d'un bien. Nous nous intéresserons plus spécifiquement à ce dernier point comme arène propice aux émotions à partir d'exemples de monuments présentant des éléments contextuels et matériels comparables. L'explosion en 2001 des bouddhas de Bâmiyân², en Afghanistan, par les talibans, est restée, dans la mémoire mondialisée, un archétype contemporain de la violence physique, idéologique et acharnée par des groupes armés à l'égard d'un bien patrimonial séculaire. Comme le rappelle Pierre Centlivres (2009) dans un numéro de revue consacré aux «Émotions patrimoniales » :

\footnotetext{
«Le 14 mars 2001, les talibans annoncent la destruction complète des bouddhas de Bamiyan et des statues du Musée national afghan, ainsi que de celles des autres musées d'Afghanistan. La destruction, disent-ils, a nécessité une semaine d'efforts et de grands moyens pyrotechniques, l'artillerie, des explosifs, entre autres. [...] la tête [du petit bouddha] avait été pulvérisée, et un tir de roquette ouvrit un trou béant à la place du bas-ventre. »
}

De même, la destruction du temple de Bêl, à Palmyre3, exposé à l'IMA, s'inscrit dans un processus analogue de " mise à mort » du monument, bien que situé dans un périmètre géographiquement éloigné. Cet acte est rapidement qualifié de « crime de guerre » dans un communiqué d'Irina Bokova (2015), directrice générale de l'Unesco (2009-2017), qui précise que le temple de Bêl est " l'un des monuments les plus emblématiques de ce site du patrimoine mondial, [et il] a été détruit le 30 août dernier [2015] au moyen d'explosifs ». Le monument détruit change instantanément de statut, il devient une ruine « violente » ou « traumatique » qui, selon Antoine Leblanc (2010 : 254), est le fruit " d'aléas violents, guerres, tremblements de terre, mais aussi explosions accidentelles, inondations, incendie, entre autres ». Des cicatrices vives et visibles pour les habitants, les communautés et les professionnels qui sont brutalement pris à témoin in situ de la perte d'un ou de plusieurs biens. Ces blessures et traumatismes violents sont autant d'indicateurs émotionnels et physiques du désordre social et culturel qui caractérisent un choc traumatique. Ce dernier prend alors place dans la mémoire collective selon deux stratégies divergentes pour Antoine Leblanc : soit conserver le souvenir de l'horreur comme cela a été le cas après la Seconde Guerre mondiale, soit l'oubli. Et l'auteur de préciser :

\section{« [...] les populations traumatisées désirent le plus souvent effacer la ruine, soit en reconstruisant à l'identique le bâtiment détruit, soit en passant le plus rapidement possible à autre chose, en construisant un nouveau bâtiment, quitte à l'associer à un mémorial » (ibid., 258).}

$4 \quad$ Lorsque ces ruines traumatiques sont exposées au musée, ces dernières questionnent les liens intimes qui lient les visiteurs aux monuments, mais aussi les stratégies expographiques mises en œuvre pour faire témoigner un site archéologique vandalisé et meurtri par la guerre, tout en donnant à voir les blessures, les cicatrices et les plaies vives inhérentes à l'histoire humaine. Dans la section suivante, nous tentons de comprendre les mécanismes scéniques à l'œuvre qui déclenchent de l'émotion chez le visiteur dans son parcours de visite.

\section{La « ruine traumatique » expographiée à I'IMA}


$5 \quad$ Nous qualifions un site monumental ayant subi in vivo les affres de la guerre de ruine « violente » ou « traumatique » comme un terreau émotionnel exacerbé par un écosystème patrimonial de nature " positive » ou " négative ", selon Nathalie Heinich (2012, 2013). L'auteure précise que les émotions sont des révélateurs de valeurs, notamment lorsqu'un monument subit un dommage violent provoquant indignation, choc ou émoi de la part des témoins directs (habitants, archéologues, touristes, etc.) ou indirects (Unesco, États, etc.) de cet événement traumatisant :
«C'est ainsi que nul ne s'étonnera qu'on puisse être indigné ou bouleversé par la destruction d'une église romane, même si tout le monde ne partage pas le goût pour l'art roman ou l'intérêt pour les lieux de culte chrétiens : c'est qu'une telle émotion (performance émotionnelle), même en l'absence d'un jugement de valeur expressément exprimé (performance axiologique), révèle la valeur positive collectivement attachée à l'ancienneté et, éventuellement, à la beauté ou encore à la signification religieuse d'un bâtiment (compétence axiologique) » (Heinich, $2012: 21)$.

Ainsi, l'émotion patrimoniale dite " positive ", à l’instar de Nathalie Heinich, relève de l'admiration que l'on témoigne à un bien ; à l'inverse, elle est dite " négative » lorsqu'elle suscite l'indignation, fruit d'émotions défensives pour son maintien à l'identique, par exemple. Cette ébauche typologique des émotions patrimoniales proposée par Heinich souligne l'attachement affectif lié à l'objet patrimonial. Elle précise, par ailleurs, que l'émotion circule autour de postures d'acteurs (la victime, l'accusateur, l'accusé, le juge) qui activent ces jeux affectifs dans l'arène sociale et expographique. Les sites antiques violentés, tels que Palmyre, et présentés aux visiteurs via un média exposition relèvent selon nous de cette circulation de jeux d'acteurs : une victime patrimoniale d'attaques terroristes (site monumental), défendue par une pluralité d'accusateurs qui dénoncent les actes violents (Unesco, journalistes, archéologues, habitants, musées, etc.) face à un accusé (État islamique) devant un juge (l'opinion publique, les visiteurs d'une exposition en France). Cet ensemble " d'actants » induit pour le visiteur un cadre déclencheur d'émotions propice aux stratégies scéniques d'une expographie immersive. Pour Dominique Gélinas (2014), l'expographie immersive se caractérise, entre autres, par le fait que le visiteur n'a plus de distanciation avec l'objet « [n]on seulement de manière physique, [il] déambule réellement dans le décor, mais également de manière psychologique. Il s'engage émotionnellement en fouillant dans son savoir et ses souvenirs propres ». Autrement dit, il n'est plus un simple visiteur, mais un acteur engagé par l'expérience de visite qui le conduit à regarder, ressentir et vivre intensément ce qui lui est donné à voir. Notre propos s'appuie sur l'exposition, Cités millénaires. Voyage virtuel de Palmyre à Mossoul 4 qui, selon le discours institutionnel, souhaite informer les visiteurs des violences massives infligées tant aux villes syriennes (Palmyre, Alep), libyennes (Leptis Magna) et irakiennes (Mossoul) qu'aux monuments, populations locales et professionnels de musées ou de sites archéologiques. Les concepteurs et scénographes de l'exposition ont choisi d'introduire le numérique dans les salles grâce à des écrans de très grandes dimensions diffusant des images captées par des drones puis modélisées et projetées en $3 \mathrm{D}$ selon une technologie développée par la société Iconem ${ }^{5}$. Le parti pris expographique repose sur une imagerie urbaine en mouvement (fig. 1) qui reprend les codes architecturaux de la ville arabe en ruine (Mossoul, Alep). À cela se superpose l'espace urbain " authentique » reconstruit à partir d'images d'archives, de photographies et d'images $3 \mathrm{D}$ dans le but de faire expérimenter aux visiteurs par les sens (vue, ouie) l'ambiance urbaine en temps de paix. Grégoire Chelkoff questionne le concept d'ambiance urbaine selon une approche immersive. Il la définit comme « une expérience [qui consiste à] placer l'éprouver au centre de notre attention, soumettre une situation, plus qu'un objet, à l'épreuve corporelle et sensible, et faire de cette épreuve un moment d'observation et d'analyse » (Chelkoff, 2018). Nous faisons donc l'hypothèse que ce prisme scénique a pour objectif d'attirer l'attention visuelle du visiteur sur l'espace urbain en mouvement (Bailly, 2018) qui consiste en un vecteur émotionnel, lui-même alimenté par l'image et l'ambiance sonore, le tout prisonnier d'un écrin mortuaire (fond d'écran noir). 
Figure 1. La ville de Mossoul (Irak) projetée sur écran et en mouvement.

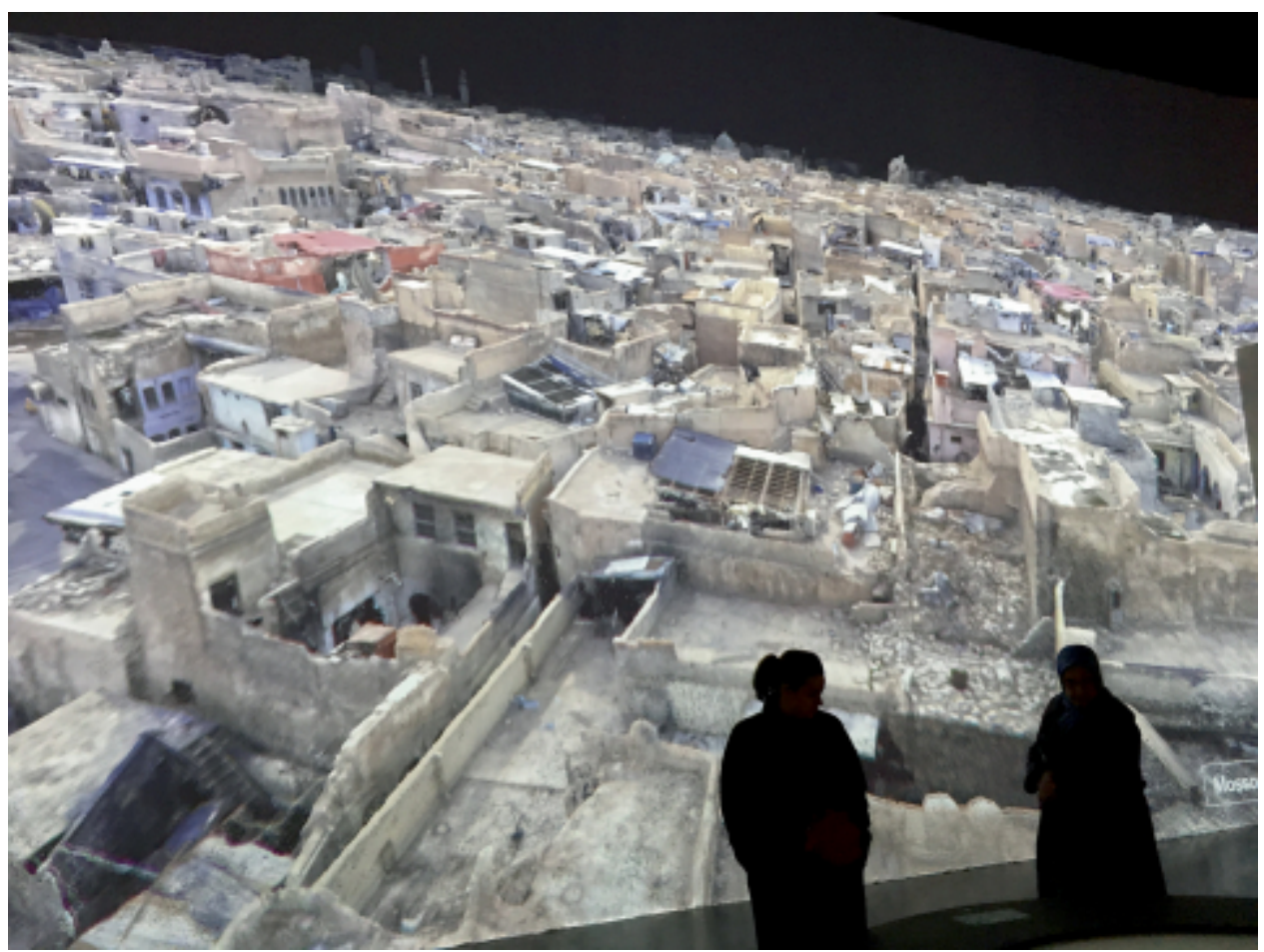

Photo : Isabelle Brianso, 2018. (C) IMA.

$7 \quad$ Les travaux d'Émeline Bailly $(2016,2018)$ montrent que le mouvement dans l'espace urbain est le socle de l'expérience sensible pour le visiteur car il « favorise les sentis multisensoriels, mais aussi les appréhensions cognitives [...] et les ressentis affectifs (via l'imagination matérielle et les émotions associées) " (Bailly, 2018). Dans ce sens, l'exposition Cités millénaires. Voyage virtuel de Palmyre à Mossoul fusionne habilement trois éléments qui animent l'espace urbain par le mouvement, à savoir : le visiteur (corps), la ville (ruines) et le monument (détruit). Cette fusion est activée par des effets scéniques et sensoriels afin de créer une vive émotion chez le visiteur. En d'autres termes, la ville devient un substrat vivant et incarné auquel le visiteur se réfère dans son intimité. Cet effet est particulièrement exprimé dans le livre d'or de l'exposition :

\footnotetext{
« Mon père est enterré en Syrie. Dieu seul sait si nous pourrons prier sur sa tombe un jour... Dieu seul sait si nous pourrons aller à nouveau à Alep, Palmyre, Homs, Hama... Une émotion magnifique » (Lana, livre d'or, janvier 2019).
}

Figure 2. Mausolée détruit à Mossoul (Irak). 


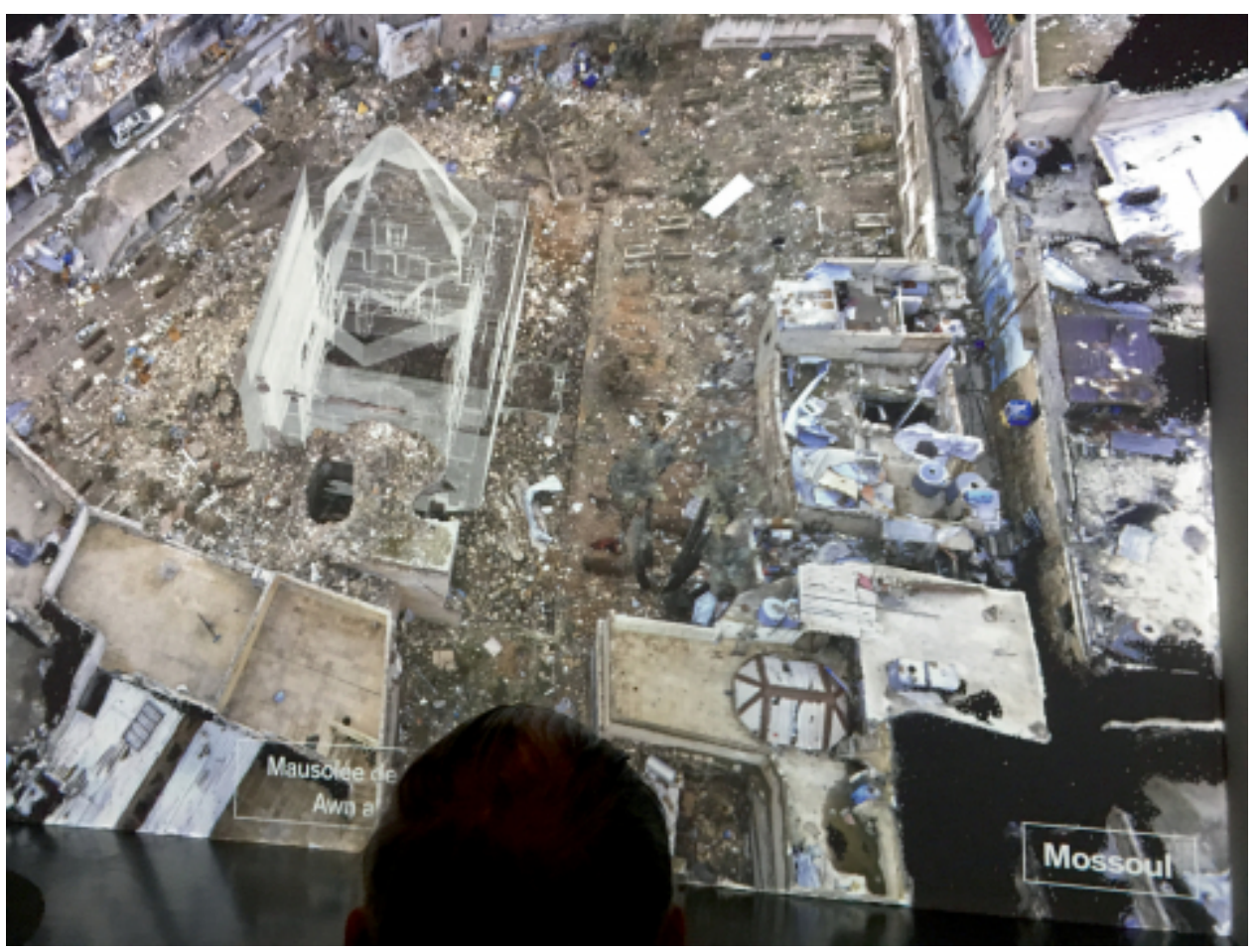

Photo : Isabelle Brianso, 2018. () IMA.

8 Par ailleurs, les images filmées projetées sur écrans construisent une ambiance mortuaire où certains monuments violentés surgissent numériquement de ruines urbaines photographiées (fig. 2), telles des apparitions dans leurs cercueils. Dans ce contexte, le visiteur se présente en tant que spectateur de ce chaos, saisi par une émotion négative au sens d'Heinich (2012), que nous définissons comme un sentiment de choc, de stupéfaction ou de sidération éprouvée devant une scène violente. Ainsi, le choix iconographique d'exposer l'espace urbain éventré (ville détruite, monument numérique) a pour objet de susciter l'indignation des visiteurs devant quatre ${ }^{6}$ sites emblématiques du monde arabe ayant subi des dommages patrimoniaux irréversibles, dont le site de Palmyre (fig. 3). L'effet est d'autant plus réussi que c'est bien d'un patrimoine universel au sens de l'Unesco dont il est question ici, la ruine millénaire authentique, qui constitue un corpus visuel violenté, sorte de reportage de guerre projeté sur écran dans le but de montrer à vif ce qu'il reste de ces villes ou de ces sites archéologiques aujourd'hui :

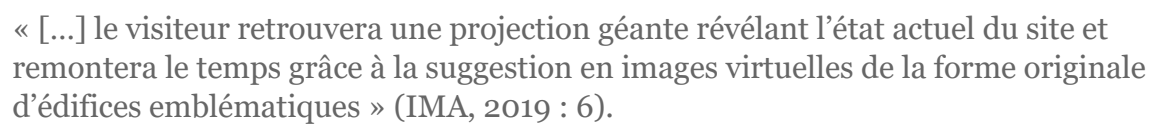

Figure 3. Temple de Baalshamîn (Palmyre, Syrie) à partir d'images numériques 3D. 


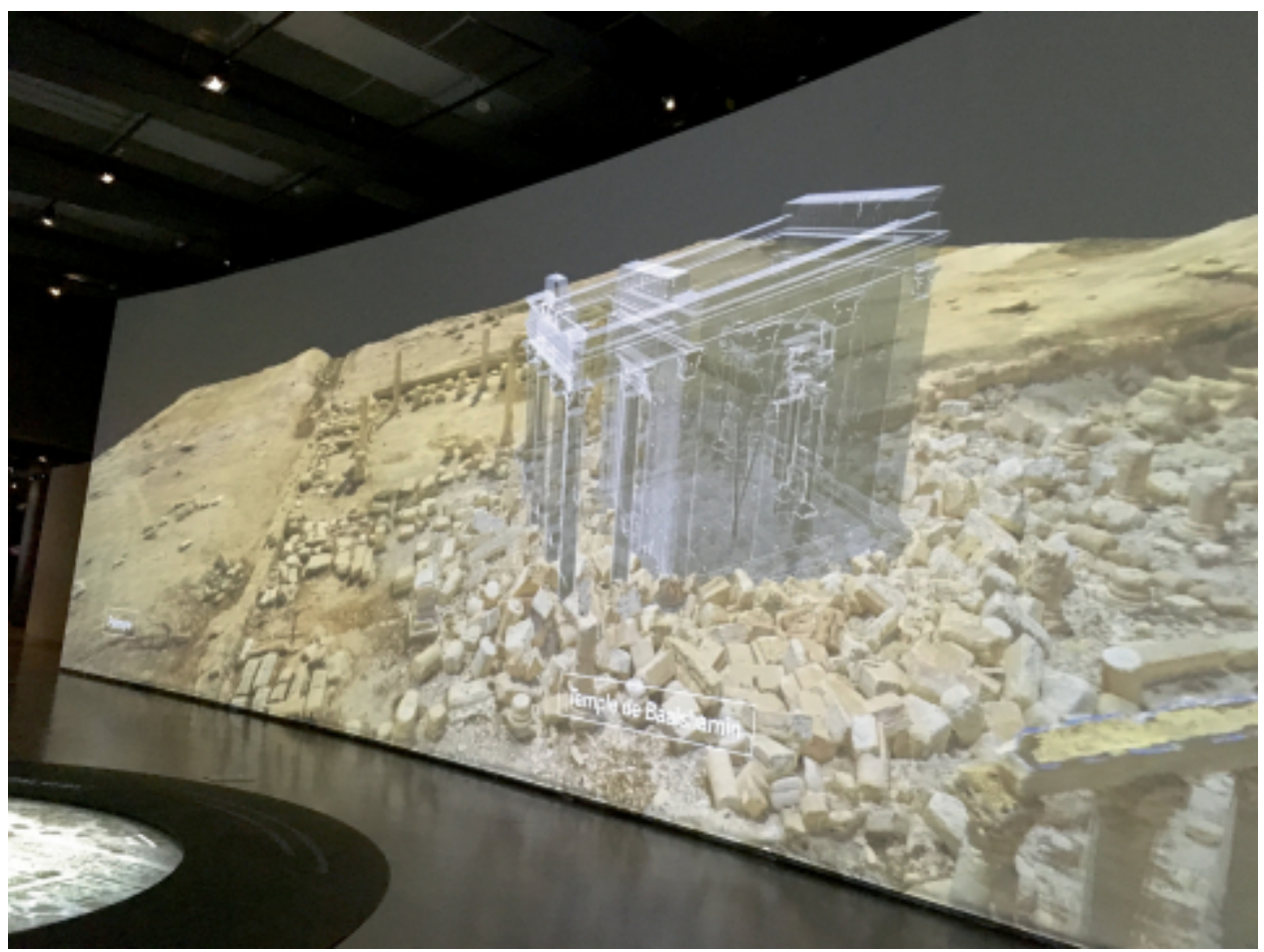

Photo : Isabelle Brianso, 2018. (c) IMA.

Du côté du visiteur, l'immersion visuelle et numérique dans l'exposition s'appuie sur une mise en scène de l'émotion urbaine grâce à la projection sur écrans d'images satellites ou d'archives montrant des bâtiments en ruines. Une immersion empathique du visiteur dans un chaos urbain où le monument numérique (fig. 3) se détache des autres ruines archéologiques afin de l'inciter à aller plus loin. Un point de vue assumé par la commissaire de l'exposition, Aurélie Clemente-Ruiz, et qui constitue un premier niveau de lecture devant engendrer un second niveau pour le visiteur...

\footnotetext{
«[...] détaché de l’image et tentant de répondre à des questions telles que : où est situé ce que je vois? Que racontent ces images ? Les grandes tables de médiation numérique installées au milieu des salles sont chargées d'apporter du contenu : la situation des bâtiments, leur date de construction, de destruction, le contexte historique... » (IMA, 2018 : 10).
}

Le visiteur expérimente donc sur écran une ambiance urbaine en situation de guerre, celle d'observer un site archéologique violenté dont seule la trace numérique est désormais lisible. Le monument violenté se présente alors selon une posture de témoin animé, errant entre deux mondes (mort, vie), et dont le récit traumatique est relayé par les habitants et les professionnels ayant tenté de protéger le site. Ces traces composent une narration mémorielle du site archéologique, chargée en émotions, à partir de témoignages relevant principalement de points de vue liés aux souvenirs. Autrement dit, la salle d'exposition consacrée au site de Palmyre utilise les souvenirs usuels d'acteurs de terrain ayant vécu au plus près des combats dans le but d'interpeler, directement et émotionnellement, les visiteurs. Nous relevons dans l'exposition trois catégories d'acteurs qui témoignent face aux visiteurs (fig. 4) selon cette posture singulière : le savant, qui relève d'une posture académique et disciplinaire (archéologie, histoire de l'art), l'expert comme professionnel de terrain (directeurs de sites et de musées, experts Unesco, Iconem) et les acteurs ordinaires (habitants, touristes) qui retracent, par le récit, les dernières années et heures du site violenté.

\section{Récits de monuments violentés : le témoignage intime et engagé des acteurs}


11 Dans cette section, nous nous intéressons à la salle consacrée au site archéologique de Palmyre qui connaît son apogée au début du IIIe siècle de notre ère et fixe son souvenir monumental grâce à un urbanisme très caractéristique, le temple de Bêl ou la grande colonnade, par exemple. Nous comprenons que dans ce contexte, les violences engendrées par l'État islamique sur ce site du patrimoine mondial (Unesco) ont suscité des émotions " négatives » d'incompréhension et d'indignation de la part des archéologues et des scientifiques du monde entier, notamment chez Paul Veyne (2015 : 10) :
« Pourquoi un groupe terroriste saccage-t-il les monuments inoffensifs d'un lointain passé (ou les met-il en vente) ? Pourquoi détruire cette Palmyre qui était inscrite par l'Unesco au patrimoine mondial de l'humanité ? Et pourquoi tant de massacres, parmi lesquels le supplice, la torture, la décapitation, le 18 août, de l'archéologue palmyrénien Khaled al-Assaad?»

L'exposition Cités millénaires. Voyage virtuel de Palmyre à Mossoul met donc en scène le récit d'une pluralité d'acteurs, comme le rappelle le discours institutionnel de l'IMA : « [...] les poignants témoignages d'archéologues, conservateurs de musée ou simples civils donnent un visage à ceux qui, au prix d'efforts parfois insensés, contribuèrent à sauvegarder ce patrimoine. » La scénographie de l'exposition joue sur les codes de la ville arabe en situation de siège pour donner à voir et à entendre des témoignages qui reposent sur une approche sensible. Nous proposons d'étudier la posture narrative de trois acteurs ou figures " témoins " (archéologue, Iconem, habitant) au moment de la destruction de Palmyre et qui jouent un rôle discursif dans le propos communicationnel de la salle consacrée au site archéologique. Tout d'abord, Michel Al-Maqdissi, ancien directeur des fouilles et études archéologiques à la Direction générale des antiquités et des musées de Syrie, fait l'objet d'un témoignage audiovisuel7 (fig. 4) diffusé en périphérie de l'espace expographique central consacré au site de Palmyre, composé d'images sur écrans et d'une table de médiation numérique.

Figure 4. Témoignage vidéo de Michel Al-Maqdissi.

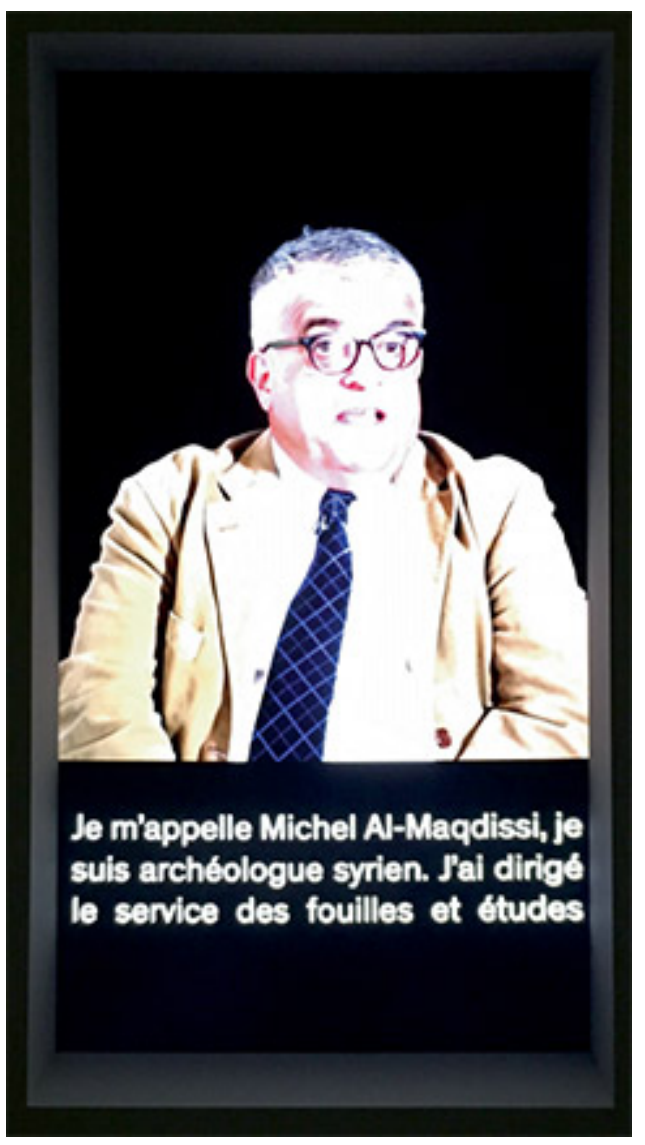

Photo : Isabelle Brianso, 2019. () IMA.

13 Cet archéologue intervient selon une posture d'expert syrien et international (en exil, il est rattaché au Musée du Louvre). Il expose aux visiteurs des opinions techniques, 
scientifiques et ses souvenirs quant au site antique de Palmyre. Ce témoignage relève d'une fabrique émotionnelle "négative » (« l'avenir du site [de Palmyre] est un avenir noir ») dont nous souhaitons comprendre les articulations discursives, vectrices d'émotions. En effet, ce témoignage est construit selon une posture de témoin exclusif au plus près des combats (« J'étais à Palmyre [...] au début des événements, en août $2015 »)$ tout en rappelant l'expertise scientifique dont Michel Al-Maqdissi est investi (" J'ai dirigé une équipe qui a fouillé plusieurs sites localisés à Palmyre et particulièrement sur l'esplanade du sanctuaire du temple de Bêl, où on a réalisé un sondage pour essayer de déterminer quelles sont les occupations qui précèdent l'actuelle phase de construction monumentale »). Cette conduite savante d'autorité induit une légitimité dans le discours énoncé tant concernant le diagnostic sanitaire du site antique que des perspectives de restauration, de conservation ou de reconstruction envisagées. Le discours s'ancre dans une sémantique de la perte due aux actes de violence (« Comment voulez-vous qu'un monument qui a été explosé à ses bases et qui est devenu de la poussière, comment voulez-vous reconstruire ce bâtiment ? »), à laquelle s'ajoute le caractère irréversible de la perte par l'impossibilité de reconstruire les monuments à l'identique ( « Toutes les propositions de reconstruction, de refaire tel ou tel monument, ne sont pas réalisables »; " On ne peut pas revenir en arrière »). Ce diagnostic que l'on peut qualifier de « pessimiste » suscite chez le visiteur une prise de conscience de la perte matérielle et mémorielle du patrimoine que nous relevons dans le livre d'or de l'exposition :

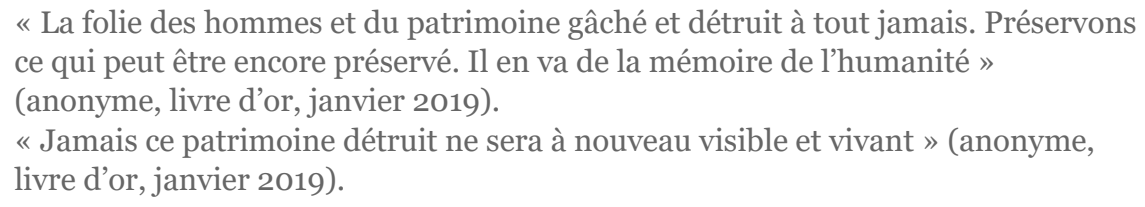

L'hypothèse scientifique de reconstruire les monuments détruits avec des matériaux modernes pourrait conduire, selon Michel Al-Maqdissi, à une hybridation du site en « Disneyland de béton ». D'autres experts ou architectes du patrimoine mondial auprès de l'Unesco, à l'instar de Pierre-André Lablaude (2018), posent la question de la reconstruction à l'identique de Palmyre dans le catalogue de l'exposition sans y apporter de réponse tranchée. Le numérique semble être un substitut face au complexe dilemme économico-politique du projet de reconstruction à l'identique. Dans ce contexte, la start-up Iconem s'érige selon une posture technologique de sauvegarde de la mémoire des monuments détruits par la production d'un " double numérique ». Iconem se positionne d'une part en tant qu'acteur de la nouvelle économie culturelle du numérique (Dufrêne et al., 2013), d'autre part en tant que réponse innovante et militante relevant d'une " citoyenneté numérique » (Greffet \& Wojcik, 2014) face aux dangers auxquels sont confrontés les sites du patrimoine mondial. Le discours institutionnel d'Iconem s'inscrit donc dans la défense du patrimoine universel confronté à des périls globaux (pillage, pollution, urbanisation, conflits armés, etc.) et dont les actions de sauvegarde ne relèveraient plus de la seule responsabilité des États ou d'institutions spécialisées (Unesco) mais de projets innovants à partir de la numérisation $3 \mathrm{D}$ de sites monumentaux à grande échelle (monument, patrimoine urbain ou rural, etc.), en somme, de biens culturels en danger délaissés par les États et la communauté internationale, malgré le travail des équipes d'archéologues. La start-up Iconem se présente donc en tant qu'acteur technologique au service de la science : la technologie utilisée (drone, photoréalisme de la $3 \mathrm{D}$ ) sert à une documentation matérielle des savoirs dans le but de lutter contre une diversité de menaces endogènes et exogènes. Un projet pilote d'exposition numérique a ainsi été lancé en décembre 2016 au Grand Palais, à Paris, intitulé Sites éternels. De Bâmiyân à Palmyre. Voyage au cœur des sites du patrimoine universel ${ }^{8}$, à partir d'images 3D immersives réalisées sur quatre sites archéologiques en danger, dont Palmyre. Un essai expographique qui n'est pas sans rappeler l'exposition à l'Institut du monde arabe et qui, au-delà d'une signature technologique de la start-up Iconem, souligne un nouveau propos communicationnel pour les sites du patrimoine mondial en péril. En effet, ces derniers souffrent d'un manque de visibilité sur la scène internationale du patrimoine 
mondialisé à côté du prestigieux label de l'Unesco qui consacre les sites remarquables de l'humanité. Cet éclairage technologique (3D) du patrimoine archéologique en danger relève d'une stratégie communicationnelle de la part de la société Iconem dont l'argumentaire repose sur le risque de disparition du patrimoine en cas de conflits armés, entretenant une atmosphère anxiogène. Cet environnement à risques conditionne le public de musée à une approche empathique face à l'urgence des actions à mener pour limiter la perte patrimoniale. Notons que l'expérience affective du visiteur est exacerbée par la réalité virtuelle du jeu vidéo d'Ubisoft ${ }^{9}$ mis à disposition des publics en fin d'exposition. Le visiteur de l'IMA se voit proposer un casque immersif avec lequel il déambule au sein de monuments violentés ayant été expographiés durant le parcours de visite. On peut alors s'interroger sur la place des habitants dans ces dispositifs numériques puisqu'ils sont absents, alors qu'ils ont été très actifs lors des actions de sauvegarde du patrimoine et restent les premières victimes de l'État islamique. Les populations locales à Palmyre semblent s'effacer au profit du monument numérisé alors même que l'émotion patrimoniale des espaces urbains violentés ne s'incarne pas uniquement à travers la figure du monument en ruine, mais également par le quotidien bouleversé des habitants en tant qu'acteurs ordinaires. Plusieurs auteurs soulignent l'intérêt d'étudier le pratiquant ordinaire en recherche. Selon Anne Watremez (2008), il est un individu (habitant, amateur) qui vit principalement dans le centre historique, détaché de toute activité touristique. Son rythme au quotidien relève néanmoins d'une certaine "banalité » que l'habitant pratique normalement dans l'espace urbain pour se rendre à son travail, faire ses courses ou encore lors de promenades familiales ou individuelles. Il n'en reste pas moins un acteur-témoin de premier plan qui habite et vit la ville ou " pratique des lieux ». Les populations locales expérimentent donc la ville par le fait du « quotidien » qui fabrique du sens par les pratiques, les mémoires, les odeurs ou encore les circulations individuelles ou collectives dans l'espace public. En d'autres termes, elles développent un point de vue vis-à-vis de la ville qui en temps de guerre revêt un caractère affectif et d'attachement lié aux souvenirs. Comme indiqué plus haut, l'exposition Cités millénaires. Voyage virtuel de Palmyre à Mossoul reprend les stéréotypes de la ville orientale, faisant notamment témoigner les habitants grâce à un espace public " authentique » de l'organisation marchande au Moyen-Orient : le souk. Ce marché traditionnel constitue " un réseau dense de rues orthogonales au centre de la cité, où se regroupent des activités commerciales séparées par des espaces domestiques » (David, $2018: 59$ ), qui symbolise l'ambiance authentique de la ville arabe pour le touriste occidental (Girard, 2006). D'autre part, le souk constitue un patrimoine culturel immatériel (artisanat) qui relie l'habitant ordinaire aux lieux du quotidien, vecteur de patrimonialité au sens où l'individu « doit permettre de comprendre la dimension de l'attachement, dimension que l'on peut aussi désigner comme la construction du regard patrimonial » (Watremez, 2008 : 13). Le témoignage intime des acteurs locaux circule dans le discours expographique (vidéo, texte) par une ambiance locale réinterprétée, issue de documents d'archives, ce qui forge chez les visiteurs l'idée d'une " orientalisation » occidentale de la ville arabe et de ses habitants. Ce constat questionne ainsi la place réelle laissée aux habitants en tant que témoins intimes de pratiques ordinaires, eu égard aux monuments violentés et ce que leurs destructions partielles ou totales ont suscité comme émotions patrimoniales. L'exposition ne répond pas de manière explicite à ce questionnement, elle utilise plutôt les stéréotypes de la ville orientale pour interpeler émotionnellement les visiteurs quant aux destructions de sites emblématiques du monde arabe et la résilience fantasmée de ses habitants face aux monuments violentés.

\section{Conclusion}

15 L'article a étudié les stratégies expographiques mises en œuvre dans la fabrique des émotions patrimoniales déclenchées face aux violences faites aux monuments en situation de conflits armés. Dans ce contexte, le monument est bien plus qu'une simple 
lecture historique et esthétique d'un site archéologique, il constitue une trace productrice de savoirs qui documente et nourrit la mémoire collective au-delà de l'objet matériel, sorte de "substrat sensible » propice aux liens affectifs. Construit pour résister aux vicissitudes du temps, notamment aux guerres et invasions diverses, de nombreux monuments millénaires disparaissent pourtant en quelques instants sous les explosifs des milices, transformant le site en champ de ruines. Dès lors, les monuments sont perçus en Occident comme des victimes civiles de la violence humaine, ce qui leur confère un nouveau statut patrimonial, celui de " ruines traumatiques ». Cette qualification définit selon nous un terreau affectif autour de l'objet patrimonial, dans lequel circulent des émotions " positives » et " négatives » produites dans l'arène sociale, puis réinterprétées dans l'exposition. Le monument antique passe donc de l'admiration collective (émotion positive) à l'indignation collective (émotion négative) selon les points de vue proposés aux publics des musées. L'exposition immersive présentée à l'Institut du monde arabe illustre cela en interpellant le visiteur par l'émotion, c'est-à-dire en lui donnant à voir et à ressentir les violences faites aux monuments. Le visiteur a donc une double posture, celui de spectateur du chaos et de témoin des violences patrimoniales en situation de siège. Son expérience de visite est délibérément chargée en émotions car elle relève d'une succession de sentiments intimes (choc, indignation) produits par des effets scéniques (image, son, mouvement) et des récits d'acteurs ayant été vécus au plus près des combats. En fin de parcours, le visiteur est informé des violences infligées aux monuments et ne peut plus les ignorer. L'article pointe, par ailleurs, d'autres bouleversements émotionnels du quotidien ordinaire des habitants (lieux de vie éventrés, repères socio-culturels désorganisés). Pourtant, le discours des habitants est peu présent dans l'exposition en comparaison du discours d'experts en exil (archéologue) ou de la technologie développée par la start-up Iconem. Des points de vue expographiques ambivalents qui questionnent (sans y répondre) l'attachement ordinaire des habitants à leurs monuments et leurs émotions devant ces « ruines traumatiques».

\section{Bibliographie}

Andrieux (Jean-Yves) \& Chevallier (Fabienne). 2014. Le Patrimoine monumental. Sources, objets et représentations. Rennes : PUR.

Bailly (Émeline). 2016. « Les paysages urbains en mal d'émotions ». Carnets de géographes [en ligne], 9 : http://journals.openedition.org/cdg/594 [consulté le 5 juillet 2020].

DOI : $10.4000 /$ cdg. 594

Bailly (Émeline). 2018. "Sentir le mouvement, éprouver la ville ». VertigO [en ligne], 18(3) : http://journals.openedition.org/vertigo/22551 [consulté le 30 juin 2020].

DOI : $10.4000 /$ vertigo.22551

Centlivres (Pierre). 2009. "Vie, mort et survie des Bouddhas de Bamiyan (Afghanistan)». Livraisons de l'histoire de l'architecture [en ligne], 17, «Émotions patrimoniales I », p. 13-26 : https://journals.openedition.org/lha/200 [consulté le 30 juin 2020].

DOI : $10.4000 /$ lha.200

Chelkoff (Grégoire). 2018. « Expérimenter l'ambiance par l'architecture ». Ambiances [en ligne], 4 : http://journals.openedition.org/ambiances/1558 [consulté le 30 juin 2020].

Choay (Françoise). 1996. L’Allégorie du patrimoine. Paris : Seuil.

Davallon (Jean). 2006. Le Don du patrimoine : Une approche communicationnelle de la patrimonialisation. Paris : Hermes Science - Lavoisier.

Davallon (Jean). 2015. " Mémoire et patrimoine : pour une approche des régimes de patrimonialisation » in Mémoire et nouveaux patrimoines [en ligne] / sous la direction de Cécile Tardy et Vera Dodebei. Marseille : OpenEdition Press : https://books.openedition.org/oep/444 [consulté le 30 juin 2020].

DOI : $10.4000 / 1895.2$

David (Jean-Claude). 2018. " Le souk d'Alep, le souk al-Zarb », p. 59-61 in Cités millénaires. Voyage virtuel de Palmyre à Mossoul. Catalogue de l'exposition à l'IMA (Paris, 10 octobre 2018 - 17 février 2019). Paris : Éditions Hazan.

Dufrêne (Bernadette), Ihadjadene (Madjid) \& Bruckmann (Denis) (dir.). 2013. Numérisation $d u$ patrimoine. Quelles médiations? Quels accès? Quelles cultures? Paris : Hermann. 
Gélinas (Dominique). 2014. « Le sensorium synthétique : réflexion sur l'utilisation de l'expographie immersive numérique et muséale ». Conserveries mémorielles [en ligne], 16 : http://journals.openedition.org/cm/2000 [consulté le 30 juin 2020].

Girard (Muriel). 2006. "Imaginaire touristique et émotion patrimoniale dans la médina de Fès ». Culture \& Musées, 8, p. 61-90.

DOI : 10.3406/pumus.2006.1405

Greffet (Fabienne) \& Wojcik (Stéphanie). 2014. « La citoyenneté numérique : perspectives de recherche ». Réseaux, 184-185, p. 125-159.

Heinich (Nathalie). 2012. "Les émotions patrimoniales : de l'affect à l'axiologie ». Social Anthropology / Anthropologie sociale, 20(1), p. 19-33.

DOI : 10.1111/j.1469-8676.2011.00187.x

Heinich (Nathalie). 2013. " Esquisse d'une typologie des émotions patrimoniales », p. 195-210 in Émotions patrimoniales / sous la direction de Daniel Fabre. Textes réunis par Annick Arnaud. Paris : Éditions de la Maison des sciences de l'homme (Cahiers d'ethnologie de la France, 27).

IMA. 2018. "Cités millénaires. Voyage virtuel de Palmyre à Mossoul ». L'Actualité de l'IMA, septembre-décembre, p. 6-10.

Kott (Christina). 2013. " Guerre et patrimoine. L'"Exposition des œuvres d'art mutilées" de 1916 », p. 119-145 in Émotions patrimoniales / sous la direction de Daniel Fabre. Textes réunis par Annick Arnaud. Paris : Éditions de la Maison des sciences de l'homme (Cahiers d'ethnologie de la France, 27).

Lablaude (Pierre-André). 2018. "Reconstruire Palmyre ? », p. 78-83 in Cités millénaires. Voyage virtuel de Palmyre à Mossoul. Catalogue de l'exposition à l'IMA (Paris, 10 octobre 2018 - 17 février 2019). Paris : Éditions Hazan.

Leblanc (Antoine). 2010. " La conservation des ruines traumatiques, un marqueur ambigu de l'histoire urbaine ». L'Espace géographique, 39(3), p. 253-266.

Rautenberg (Michel). 2008. « Du patrimoine comme œuvre au patrimoine comme image », p. 918 in Stratégies identitaires de conservation et de valorisation du patrimoine / sous la direction de Jean-Claude Nemery, Michel Rautenberg et Fabrice Thuriot. Paris : L'Harmattan (Administration et aménagement du territoire).

Riegl (Aloïs). 1984. Le Culte moderne des monuments. Paris : Seuil.

DOI : $10.4000 /$ socio-anthropologie.5

Unesco. 2015. « La directrice générale Irina Bokova manifeste sa profonde consternation suite à la destruction du temple de Bêl à Palmyre ". Communiqué de presse du 1er septembre 2015, en ligne : https://fr.unesco.org/news/directrice-generale-irina-bokova-manifeste-sa-profondeconsternation-suite-destruction-du [consulté le 30 juin 2020].

Unesco. 2015. « La directrice générale de l'Unesco condamne la destruction de l'arc de triomphe de Palmyre : "Les extrémistes ont peur de l'Histoire" ». Communiqué de presse du 5 octobre 2015, en ligne : https://fr.unesco.org/news/directrice-generale-unesco-condamne-destructionarc-triomphe-palmyre-extremistes-ont-peur [consulté le 30 juin 2020].

Veyne (Paul). 2015. Palmyre, l'irremplaçable trésor. Paris : Albin Michel.

Watremez (Anne). 2008. "Vivre le patrimoine urbain au quotidien : pour une approche de la patrimonialité ». Culture \& Musées, 11, p. 11-36.

DOI : 10.3406/pumus.2008.1467

Waty (Bérénice). 2016. «Émotions patrimoniales. Quand le patrimoine devient l'affaire de tous ». Bulletin des bibliothèques de France (BBF), 7, p. 8-22.

\section{Notes}

1 Définition donnée par le Trésor de la langue française informatisé, en ligne : http://atilf.atilf.fr (consulté le 30 juin 2020).

2 Le paysage culturel et les vestiges archéologiques de la vallée de Bâmiyân ont été inscrits sur la liste du patrimoine mondial de l'Unesco en 2003.

3 Le site de Palmyre a été inscrit sur la liste du patrimoine mondial de l’Unesco en 1980.

4 Exposition temporaire (10 octobre 2018 - 10 février 2019) présentée à l'Institut du monde arabe (IMA), à Paris, en partenariat avec l'Unesco.

5 Start-up fondée en 2013 et spécialisée en numérisation 3D des sites patrimoniaux dans le monde.

6 Palmyre, Alep, Mossoul et Leptis Magna.

7 Témoignage de quatre minutes produit à Paris en 2018 par l'IMA. Témoignage accessible grâce à un écouteur circulaire accroché au mur.

8 Exposition immersive organisée par la Réunion des musées nationaux, le Grand Palais et le Musée du Louvre du 14 décembre 2016 au 9 janvier 2017. 


\section{Table des illustrations}

Titre Figure 1. La ville de Mossoul (Irak) projetée sur écran et en mouvement.

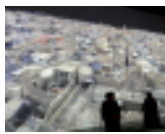

Crédits Photo : Isabelle Brianso, 2018. (C) IMA.

URL http://journals.openedition.org/culturemusees/docannexe/image/5391/img1.jpg

Fichier image/jpeg, 1,1M

Titre Figure 2. Mausolée détruit à Mossoul (Irak).

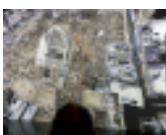

Crédits Photo : Isabelle Brianso, 2018. ( IMA.

URI http://journals.openedition.org/culturemusees/docannexe/image/5391/img2.jpg

Fichier image/jpeg, 1,3M

Titre Figure 3. Temple de Baalshamîn (Palmyre, Syrie) à partir d'images

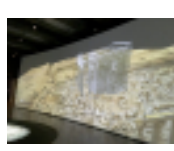

Crédits Photo : Isabelle Brianso, 2018. (c) IMA.

URI http://journals.openedition.org/culturemusees/docannexe/image/5391/img3.jpg

Fichier image/jpeg, 1,1M

Titre Figure 4. Témoignage vidéo de Michel Al-Maqdissi.

Crédits Photo : Isabelle Brianso, 2019. ㄷ IMA.

URL http://journals.openedition.org/culturemusees/docannexe/image/5391/img4.jpg

Fichier image/jpeg, 33k

\section{Pour citer cet article}

Référence papier

Isabelle Brianso, « Le monument violenté : de l'émotion à la mise en exposition », Culture \& Musées, 36 | 2020, 35-56.

Référence électronique

Isabelle Brianso, « Le monument violenté : de l'émotion à la mise en exposition », Culture \& Musées [En ligne], 36 | 2020, mis en ligne le 23 novembre 2020, consulté le 28 mai 2021. URL: http://journals.openedition.org/culturemusees/5391; DOI :

https://doi.org/10.4000/culturemusees.5391

\section{Auteur}

\section{Isabelle Brianso}

Avignon Université, Centre Norbert Elias

Isabelle Brianso est maîtresse de conférences à l'université d'Avignon, Centre Norbert Elias (UMR 8562), où elle enseigne la muséologie et les sciences de l'information et de la communication appliquées au patrimoine culturel. Ses travaux de recherche portent sur les discours et l'analyse des processus de patrimonialisation dans un contexte de labellisation internationale (Unesco, Conseil de l'Europe), ainsi que sur l'étude des visiteurs sino-asiatiques au musée. Parmi ses publications récentes, on peut citer « L'expérience de visite des touristes chinois à Versailles : entre stéréotype et représentations " paru dans la revue Communication \& Langages ( $\left.n^{\circ} 191,2017\right)$. Elle a également coordonné avec Françoise Rigat (Université de la Vallée d'Aoste, Italie) le $n^{\circ} 33$ de Culture \& Musées (2019) sur la fabrique des patrimoines européens au XXIe siècle. Ancienne boursière Marie Curie (Intra-European Fellowship, FP7) à l'Université autonome de Barcelone (Espagne), elle est aujourd'hui membre élue au comité français du Conseil international des musées (ICOM).

Courriel : isabelle.brianso[at]univ-avignon.fr

Articles du même auteur

Maria Gravari-Barbas. 2020. Le Patrimoine mondial. Mise en tourisme, mise en images

[Texte intégral disponible en juin 2021]

Paru dans Culture \& Musées, 37 | 2021 
Introduction [Texte intégral]

Paru dans Culture \& Musées, 33 | 2019

Éditorial [Texte intégral]

Paru dans Culture \& Musées, $31 \mid 2018$

\section{Droits d'auteur}

Culture \& Musées 